de Molon Rafael Scaf (Orcid ID: 0000-0003-1110-6233)

\title{
Characterization of ligature-induced experimental periodontitis
}

Rafael Scaf de Molon ${ }^{1}$, Chan Ho Park ${ }^{2}$, Qiming Jin ${ }^{3}$, Jim Sugai ${ }^{4}$, Joni Augusto Cirelli ${ }^{1}$

${ }^{1}$ Department of Diagnosis and Surgery, School of Dentistry at Araraquara, Sao Paulo State University - UNESP. Araraquara, SP, 14801-903, Brazil

${ }^{2}$ Dental Research Institute, School of Dentistry, Seoul National University, Seoul, 08826, Republic of Korea

${ }^{3}$ Department of Cariology, Restorative Sciences and Endodontics, School of Dentistry, University of Michigan, Ann Arbor, MI, 48109-1078, USA

${ }^{4}$ Department of Periodontics and Oral Medicine and Center for Craniofacial Regeneration, School of Dentistry, University of Michigan, Ann Arbor, MI, 48109-1078, USA

Running Title: Ligature and alveolar bone loss in rats

\section{Corresponding Author:}

Dr. Joni Augusto Cirelli, Ph.D

Department of Diagnosis and Surgery, School of Dentistry at Araraquara

Sao Paulo State University - UNESP

This is the author manuscript accepted for publication and has undergone full peer review but has not been through the copyediting, typesetting, pagination and proofreading process, which may lead to differences between this version and the Version of Record. Please cite this article as doi: $10.1002 /$ jmet.23101

This article is protected by copyright. All rights reserved. 
Rua Humaita, 1680; 14801-903. Araraquara, Sao Paulo, Brazil.

Tel/Fax: +55 (16) 3301-6375

Email: cirelli@foar.unesp.br

\begin{abstract}
We sought to better characterize the progression of periodontal tissue breakdown in rats induced by a ligature model of experimental periodontal disease (PD). A total of 60 male Sprague-Dawley rats were evenly divided into an untreated control group and a PD group induced by ligature bilaterally around first and second maxillary molars. Animals were sacrificed at 1, 3, 5, 7, 14, and 21-days after the induction of PD. Alveolar bone loss was evaluated by histomorphometry and microcomputed tomography ( $\mu \mathrm{CT})$. The immune-inflammatory process in the periodontal tissue was assessed using descriptive histologic analysis and quantitative polymerase chain reaction (qPCR). This ligature model resulted in significant alveolar bone loss and increased inflammatory process of the periodontal tissues during the initial periods of evaluation (0-14 days). A significant increase in the gene expression of proinflammatory cytokines, interleukin-1 $\beta$ (IL-1 $\beta$ ), interleukin-6 (IL-6), and tumor necrosis factor- $\alpha$ (TNF- $\alpha$ ), and proteins involved in osteoclastogenesis, receptor activator of nuclear factor-k B ligand (RANK-L) and osteoprotegerin (OPG) was observed in the first week of analysis. In the later periods of evaluation (14-21 days), no significant alterations were noted with regard to inflammatory processes, bone resorption, and expression of cytokine genes. The ligature-
\end{abstract}


induced PD model resulted in progressive alveolar bone resorption with two different phases: Acute (0-14 days), characterized by inflammation and rapid bone resorption, and chronic (14-21 days) with no significant progression of bone loss. Furthermore, the gene expression of IL-6, IL-1 $\beta$, TNF- $\alpha$, RANK-L and OPG were highly increased during the progress of PD in the early periods.

Key Words: Periodontal disease; rats; periodontitis; alveolar bone loss; bone resorption; X-ray microtomography.

\section{Research Highlights}

- Ligature-induced bone resorption in rats occurred in the initial periods after disease induction;

- The bone resorption was characterized by two distinct phases: Acute (0-14 days), with pronounced inflammation and alveolar bone loss;

- Chronic phase (14-21 days): No further disease progression;

- Several proinflammatory cytokines were increased during the progress of periodontitis.

This article is protected by copyright. All rights reserved. 


\section{INTRODUCTION}

Periodontal disease, an infection condition of the supporting tissues around the teeth, is caused by an accumulation of dental biofilm and subsequent release of proinflammatory mediators, cytokines, growth factors and signaling molecules. The persistent presence of oral microorganisms to the dental structures lead to weakened periodontal tissue causing an imbalance in bone metabolism, resulting in substantial alveolar bone resorption (Alencar et al., 2002; de Molon et al., 2016b; Mizuno et al., 2015). Recent observations in adult humans identify a prevalence of periodontal disease varying around 46\%, which represents 64.7 million people with periodontitis in the United States population (Eke et al., 2015), and is considered the most common cause of tooth loss (Albandar and Rams 2002).

This article is protected by copyright. All rights reserved. 
Uncoupling of bone formation and resorption are associated with a higher degree of tissue breakdown during the course of periodontitis in susceptible hosts. At a histological level, periodontal disease leads to an inflammatory infiltrate composed of neutrophils and leukocytes, which activate immune cells such as lymphocytes to trigger

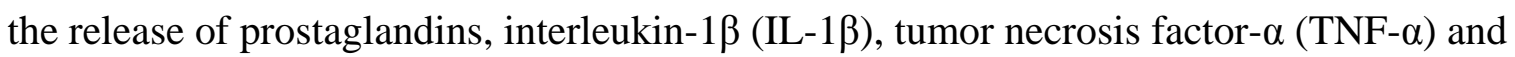
IL-6. These cellular events culminate with the activation of osteoclastogenesis and bone destruction through a direct stimulation of osteoclasts or through the release of tissuedestructive enzymes by the inflammatory cells (Mundy 1991).

Paralleling clinical observations of periodontal disease, histological and molecular analysis of experimental animal models of periodontitis in mice and rats have provided important insights into the disease pathogenesis recapitulating clinical, radiographic and histologic features of the disease (de Aquino et al., 2014; de Molon et al., 2014b; de Molon et al., 2016b; Graves et al., 2008; Hiyari et al., 2018a; Hiyari et al., 2018b; Li and Amar 2007; Polak et al., 2013; Polak et al., 2009; Saadi-Thiers et al., 2013; Wilensky et al., 2005). Moreover, animal models with physiological complications have been extensively utilized to develop new treatment modalities with more effective therapeutic strategies, inquiry host-pathogen interactions, study the effects of surgical interventions, and evaluate the intrinsic effects of periodontal infection or inflammation on systemic conditions (de Molon et al., 2014b; de Molon et al., 2013; de Molon et al., 2016b; Graves et al., 2008; Graves et al., 2012; Matsuda et al., 2015). 
Among the various methods employed to mimic periodontitis in animals, the most commonly used model is ligature-induction (de Molon et al., 2014b; de Molon et al., 2013; de Molon et al., 2016b; Pirih et al., 2015; Wong et al., 2017). The placement of a silk ligature throughout the cervical region of mandibular or maxillary molar teeth leads to bacterial colonization, stimulating a great accumulation of biofilm, resulting in symptoms of disease similar to those observed in a clinical situation, such as apical epithelial migration and bone loss (de Molon et al., 2016b; Klausen 1991; Saadi-Thiers et al., 2013). According to previously published studies in which ligatures are used (Cavagni et al., 2016; de Molon et al., 2014b; de Molon et al., 2016b; de Souza et al., 2011; Nogueira et al., 2017; Nogueira et al., 2014), connective tissue and bone loss predictably occurs over a period of 7 to 15 days in rats and mice. This feature is due to the presence of bacterial species around and within the silk thread, which leads to increased bone destruction in the early stages of disease. A previous report (Bezerra et al., 2002) showed that ligatures did not induce significant bone resorption in germ-free rats and that bacteria accumulation around the thread played an important role in the disease progression (Matsuda et al., 2015). Furthermore, traumatic injury during the pathogenesis of the ligature model has been described in the literature, especially when mouse models are utilized (Abe and Hajishengallis 2013).

Different data from the literature that directly explore and compare the characteristics of ligature-induced periodontitis during the initial stage of disease are 
scarce. Here, our aim was to better characterize the ligature-induced periodontal disease model in rats at radiographic $(\mu \mathrm{CT})$, histologic and gene expression of putative proinflammatory cytokines in the gingival tissues to more closely investigate periodontitis progression.

\section{MATERIALS AND METHODS}

\subsection{Animal care}

This article is protected by copyright. All rights reserved. 
The experimental protocol (\#13/2007) was handled according to the guidelines of the University of Michigan Institutional Animal Care and Use Committee (IACUC), and all animals received humane care conforms to the ARRIVE (Animal Research: Reporting of In Vivo Experiments) guidelines (Kilkenny et al., 2012). Rats were kept in the animal facilities under similar conditions (humidity $60 \%$, temperature-controlled $22 \pm 2{ }^{\circ} \mathrm{C}$, and 12-hour light/dark cycle). All rats were housed in standard cages in groups of two animals, and water and food were provided ad libitum. A total of 60 two-month-old male Sprague-Dawley rats, with average body weights between 180 and 200 g were randomly, by means of a raffle method, divided into two groups: an untreated control group and a ligature-induced periodontal disease group.

\subsection{Induction of periodontal disease}

All animals in the ligature-induced group were anesthetized with an inhalation method using isoflurane (Baxter Healthcare, Deerfield, IL, USA) for periodontal disease induction. The ligatures were placed using a sterilized silk thread (Ethicon, Johnson \& Johnson, Somerville, NJ) tied around the cervix of the first and second maxillary molars in an "8" shape, knotted at the palatal surface of the second molar (Fig. 1A-C). To facilitate ligature placement, a slightly separation in the interproximal area between $1^{\text {st }}$ and $2^{\text {nd }}$ molars with the aid of a periodontal probe (Hu- Friedy, Chicago, IL, USA) was performed. In all animals, the ligature was inspected every other day and repositioned if 
necessary to maintain the ligature during the entire experimental period. Ligatures were maintained in position during the whole experimental period.

\subsection{Maxillae dissection and analyses}

At each time point following periodontitis induction, five rats each from the control and experimental group were euthanized per $\mathrm{CO}_{2}$ overdose. The maxilla from each animal was dissected and then hemisected. One half of each maxilla sample was submitted for alveolar bone level measurements via micro-computed tomography $(\mu \mathrm{CT})$, after which these were utilized for routine histologic processing for descriptive histological analysis. For the other half maxilla, the gingival tissues around the $1^{\text {st }}$ and $2^{\text {nd }}$ maxillary molars were gently removed for extraction of total RNA for reverse transcription and qPCR. Following gingival tissue dissection, bone samples were immersed in $3 \%$ hydrogen peroxide for $24 \mathrm{~h}$ to facilitate the removal of all remaining soft tissue. Samples were then kept in ethanol (70\%) until its use for macroscopic evaluation of bone loss.

\subsection{Macroscopic analysis of bone resorption}

The bone resorption area in the buccal and palatal surface of the $1^{\text {st }}$ and $2^{\text {nd }}$ maxillary molars was macroscopically measured. Briefly, bone samples were removed from the $70 \%$ ethanol, dried, submerged for $5 \mathrm{~min}$ in methylene blue $(0.7 \mathrm{~g} / \mathrm{L})$. The samples were then washed with water to eliminate the additional methylene blue stain. Digital photographs of the buccal and palatal surfaces of the stained samples were obtained with a stereomicroscope (Leica Microsystems, Wetzlar, Germany) (20x magnification). The 
distance from the cemento-enamel junction (CEJ) to the alveolar bone crest (ABC) was measured at 6 different sites per tooth (mesio-distal, disto-mesial, buccal-palatal and palatal-buccal regions) and mean values were used. Images from methylene blue-stained samples were measured using appropriate software for 2D-image analysis (Image ProPlus 5.1, Rockville, MD, USA) by a blinded and calibrated examiner (JAC), as previously described (de Souza et al., 2011). Measurements were performed at baseline, and 7, 14 and 21 days after periodontal disease induction.

\subsection{Micro-computed tomography scanning}

Dissected maxillae were carefully harvested, fixed in paraformaldehyde (4\%) for 2 days, and stored in ethanol (70\%) before $\mu \mathrm{CT}$ scanning. Bone samples were then washed in distilled water and stored in $0.9 \%$ saline solution overnight for rehydration. The maxillae were scanned with $18 \mu \mathrm{m}^{3}$ voxel-size by a high-resolution $\mu \mathrm{CT}$ imaging system (GE Healthcare, London, ON, Canada). For sample reconstruction, an analysis software (Microview Analysis+ v.2.1.2, GE Healthcare, USA) was utilized and the specific parameters used were employed as described elsewhere (Cirelli et al., 2009). The linear bone loss were evaluated by two independent and calibrated examiners, and the measurements were determined from the CEJ to the $\mathrm{ABC}$ at the palatal surface of the mesio- and disto-palatal roots of the maxillary $1^{\text {st }}$ and $2^{\text {nd }}$ molar teeth (Fig. 3), as previously described (de Molon et al., 2014a; de Molon et al., 2016a; de Molon et al., 
2015). The linear measurements resulted in an average value, which was used to define the distance from the CEJ to $\mathrm{ABC}$ in millimeters.

\subsection{Histologic processing and descriptive analysis}

After $\mu \mathrm{CT}$ scanning, the maxilla samples were decalcified in $10 \%$ ethylenediaminetetraacetic acid (EDTA), $\mathrm{pH} 8.0$ for 5-6 weeks at room temperature. Samples were paraffin-embedded and $5 \mu$ m-thick sections were obtained in the mesiodistal direction. Samples were stained with hematoxylin and eosin (H\&E) for histologic descriptive analysis and were evaluated by a blinded, and calibrated examiner.

\subsection{Quantitative PCR (qPCR)}

Total RNA was extracted from the gingival palatal tissue biopsies collected from the mesial aspect of the $1^{\text {st }}$ molar to the distal site of the $2^{\text {nd }}$ molar, as previously described (Cirelli et al., 2009). Briefly, RNA purification was achieved using a RNeasy Mini Kit complemented with RNase- Free DNase Set (Qiagen, Valencia, CA, USA), according to the manufacturer's instructions. Then, a spectrophotometer (BioMate 3, Rochester, NY, USA) was used to measured the purity and quantity of total RNA by evaluating the absorbance at $260 \mathrm{~nm}$ and the 260/280 $\mathrm{nm}$ ratios, respectively. After the confirmation of the total RNA integrity, cDNA was synthesized by reverse transcription (RT) of $400 \mathrm{ng}$ total RNA in a reverse transcriptase reaction (High capacity cDNA synthesis kit, Applied Biosystems), according to the manufacturer protocol. 
Real-time, qPCR was performed by means of Real Time PCR System (ABI Prism 7500 StepOne Plus, Applied Biosystems, CA, USA) using TaqMan Gene Expression Assays (Applied Biosystems, CA, USA). The reaction was performed following the manufacturer's instruction. The determination of the levels of gene expression was performed using the cycle threshold (Ct) method and normalized to the GAPDH (housekeeping gene). The results are represented as the mean \pm SD mRNA expression from triplicate measurements normalized using the internal control GAPDH, as previously described (Cirelli et al., 2009; de Molon et al., 2014b). The target gene, ABI ID no., and reporter probe sequence of each specific TaqMan Gene Expression Assay (Applied Biosystems, Foster City, CA, USA) are described in Table 1.

\subsection{Statistics}

GraphPad Prism Software (La Jolla, CA, USA) was used to analyze the raw data. Group measurements were expressed as the mean and standard deviation (SD). Statistical significance was assessed using one-way analysis of variance (ANOVA) followed by the Tukey's post-hoc test for multiple comparisons among groups to determine the presence of any significant difference. A significance level of $5 \%$ was used.

This article is protected by copyright. All rights reserved. 


\section{RESULTS}

\subsection{Macroscopic bone resorption analysis}

To investigate whether periodontal disease led to bone resorption for the entire experimental period, macroscopic bone loss measurements were performed using methylene blue-stained maxillae samples (2A-H) at baseline (no ligature) and after 7, 14 
and 21 days of periodontitis induction (Fig. 2I). Increased bone resorption was predominantly noted after 7 days of disease induction until the $14^{\text {th }}$ day period (Fig. 2I). Significant bone resorption was observed from baseline until 14 days after ligature placement in the interproximal area measured in the distal aspect of the $1^{\text {st }}$ molar $(\mathrm{p}<$ 0.001 ) and mesial aspect of the $2^{\text {nd }} \operatorname{molar}(\mathrm{p}<0.0001)$. After 21 days, statistically significant differences in bone loss were also noted when compared to the baseline and 7 day time points. Interestingly, no significant differences in linear bone loss were found between 14 and 21 days (Fig. 2I), which suggests that periodontal disease tends to stabilize during the late periods, characterizing a chronic inflammatory disease.

\subsection{Micro-computed tomography analysis}

To further characterize the progression of periodontal disease induced by ligatures, $\mu \mathrm{CT}$ scanning was performed after the end of the experimental periods and linear measurements were made (Fig. 3). To quantitate bone loss, the CEJ to ABC distance was measured (Fig. 4). No statistically significant differences were found between control and ligature groups at the mesial side of the $1^{\text {st }}$ molar, throughout the experimental periods (Fig. 4A). However, statistically significant differences in bone resorption were found at the distal side of the $2^{\text {nd }}$ molar and within the interproximal area between the $1^{\text {st }}$ and $2^{\text {nd }}$ molars, 3 days after ligature placement compared to the untreated control group $(\mathrm{p}<$ 0.05). Progressive bone resorption was evidenced until the 14-day time point, which 
represents an acute form of the disease (Fig 4D). On the other hand, a slight decrease in bone loss was noted after 15 days of ligature placement, which was sustained until the end of the experimental period of 21 days (Fig. 4B-C). This situation represents a chronic characteristic of the disease without significant alveolar bone loss progression (Fig. 4D). Statistically significant differences in bone loss were evident between the control group and the experimental group until the end of the 21-day period $(\mathrm{p}<0.05)$.

\subsection{Histological analysis}

To evaluate histologic features of periodontitis, sagittal sections were obtained in the middle of the $1^{\text {st }}$ and $2^{\text {nd }}$ molar crowns and analysis were performed in the furcation and interproximal area after a period of 1, 3 and 5 days. All untreated control rats showed normal alveolar bone and marginal epithelium, and an absence of inflammatory infiltrate (data not shown). In the experimental group, inflammatory infiltrate (black arrows) was evident 1 day after ligature placement in the furcation and in the interproximal area (Fig. 5A-C). Remarkably, bone loss was detected in the ligature group after 1 day of ligature placement represented by an increased distance from the furcation top to the ABC. These findings were maintained 3 and 5 days after disease induction. Collectively, changes in the experimental group included an intense infiltration of inflammatory cells, disrupted epithelial integrity at the dentogingival junction, connective tissue attachment loss, and 
alveolar bone resorption characterized by an increase in the distance between the CEJ to ABC (Fig. 5 A-I).

\section{4 qPCR}

To assess the expression of proinflammatory cytokines in the periodontal tissues, qPCR was performed after the end of the experimental period. As expected, significant increases in the mRNA expression of Interleukin-6, Interleukin-1 $\beta$, and Tumor Necrosis Factor- $\alpha$ were observed during the early periods of the disease; $1(\mathrm{p}<0.01)$ and $3(\mathrm{p}<$ 0.05) days after ligature placement (Fig 6A, C, E). Accordingly, the mRNA expression of receptor activator of nuclear factor-k B ligand (RANKL), and osteoprotegerin (OPG) was more pronounced in the early periods of the disease (3 and 5 days) with statistically significant differences in the expression of RANK-L and OPG after $3(p<0.05)$ and 5 days $(\mathrm{p}<0.01)$ (Fig. 6G-H). After 1 week of disease induction, statistically significant increases in the expression of IL-6 and IL-1 $\beta$ were also noted (Fig. 6B, D) $(p<0.01)$. No differences were found for TNF- $\alpha$ after 1 week of disease (Fig. 6F). In the later periods of evaluation (14-21 days), no statistically significant differences were found for all the cytokines evaluated (Fig. 6B, D, F). The ratio of RANK-L/OPG was also evaluated and increased ratio in the $3(\mathrm{p}<0.01)$ and 5 days period $(\mathrm{p}<0.05)$ after periodontitis induction was observed (Fig 6I).

This article is protected by copyright. All rights reserved. 


\section{DISCUSSION}

Major progress in the understanding of periodontitis pathogenesis has been made since experimental models of PD were first introduced in dental research. However, important gaps in our knowledge still exist related to the ligature-induced periodontitis model. A strategy to overcome these gaps is the extensive effort of research groups to better characterize animal models that closely mimic how periodontitis presents in human beings, assisting in the selection of the most appropriate animal model to be used in preclinical studies according to their objective (de Molon et al., 2014b; de Molon et al., 2016b; Graves et al., 2008; Graves et al., 2012; Hiyari et al., 2015; Hiyari et al., 2018b; Li and Amar 2007; Pirih et al., 2015; Polak et al., 2009; Saadi-Thiers et al., 2013; Wilensky et al., 2005; Wong et al., 2017).

Experimental models for periodontitis in rats and mice have been very beneficial and important to examine various biologic hypotheses in physiological complications and reproduce the radiographic, clinical, molecular and histologic features of human periodontitis (Anbinder et al., 2016; Cavagni et al., 2016; de Aquino et al., 2014; de 
Molon et al., 2014b; de Molon et al., 2016b; Graves et al., 2008; Graves et al., 2012). Animal models of PD allow for studying cellular and molecular mechanisms as well as biological mediators involved during the establishment and progression of the disease and provide valuable information on host-microbial interactions and inflammation (Anbinder et al., 2016; Cavagni et al., 2016; de Aquino et al., 2014; de Molon et al., 2014b; de Molon et al., 2016b; de Souza et al., 2011; Graves et al., 2008; Matsuda et al., 2015; Mizuno et al., 2015; Nogueira et al., 2014). At this point, ligature-induced PD has been frequently used in periodontal research due to the involvement of live microbes naturally existent in animal species with distinct virulence features including toxins, pathogen-associated molecular patterns (PAMPs) and products of the microbial metabolism (de Souza et al., 2011). However, the variability of the results found in the literature with regard to this model hinders a definitive conclusion about the true characteristics (initiation and progression) of the disease.

Therefore, using the ligature-induced periodontitis model in rats, we addressed the host response at morphometric, radiographic, histologic and molecular levels in the beginning stages of the disease through the end of the 21-day experimental period. Our results indicate that the ligature model leads to progressive alveolar bone resorption, suggesting two distinctive phases: (1) an acute phase (0-14 days) and (2) a chronic phase (15-21 days). Furthermore, the ligature-induced PD model in an " 8 " shape placed around 
the first and second maxillary molars offers a reliable model with site-specific epithelial downgrowth, inflammatory cell infiltration, and time-dependent alveolar bone resorption.

The need to more accurately reflect the clinical reality has led researchers to better characterize existing animal models (de Molon et al., 2014b; de Molon et al., 2013; de Molon et al., 2016b; Graves et al., 2008; Graves et al., 2012). In this effort, we used different methodologies to describe the ligature model specifically during the early stages of the disease. Histologic analysis revealed that 1 day after ligature placement, evidence of bone resorption in the furcation and interproximal regions were noted in the ligated animals. The distance from the furcation top to the ABC increased, which denotes increased bone loss. Since bone resorption in the ligature model is dependent on the presence of oral microorganisms (Abe and Hajishengallis 2013; Bezerra et al., 2002; Klausen 1991; Matsuda et al., 2015), this finding can be attributed to the trauma caused during the ligature placement (Abe and Hajishengallis 2013). Although $\mu \mathrm{CT}$ analysis had no statistically significant differences in bone resorption within the first 3 days of disease, 5 days after ligature placement, a statistically significant increase in bone resorption was noted radiographically for the experimental group, which increased over time until the $14^{\text {th }}$ day. Importantly, after 14 days of periodontitis a slightly decrease in the amount of bone resorption was evidenced in the interproximal and distal region of the second molar. These results suggest that ligature-induced periodontal disease leads to two distinct phases: an acute phase with progressive destruction of the periodontal tissues ( 0 to 14 
days) followed by a chronic phase characterized by a cessation of the periodontal inflammation and tissue breakdown. This profile change of ligature-induced bone loss could be potentially explained because, as a consequence of alveolar bone resorption, periodontal tissues tend to migrate to a more apical position to recuperate the biologic space, diminishing the disease severity over time (de Molon et al., 2014b; de Molon et al., 2016b). However, in this study, the ligature was displayed further apically, every 3 days, to keep the silk thread in close contact with the periodontal tissue, and consequently maintain the inflammation.

In an effort to maintain the disease severity over time, previous studies ( $\mathrm{Li}$ and Amar 2007; Saadi-Thiers et al., 2013; Yuan et al., 2011) suggest the incubation of live pathogenic microorganisms into the thread, which could lead to an exacerbation of the disease intensity. Microorganisms adhered to the ligature produce several virulence factors, such as PAMPs including some toxins, which might result in a more complex host response, affecting the profile of proinflammatory mediators, and periodontal tissue destruction (de Souza et al., 2011). Additionally, a previous study (Anbinder et al., 2016) utilized ligature placement around the first maxillary molar associated with an oral gavage model using Porphyromonas gingivalis to increase the disease severity. Furthermore, the ligature was repositioned apically to sustain the thread in intimate contact with the marginal gingiva. The authors concluded that this model was suitable when advanced bone loss is expected and maintained over time. 
Here, mRNA levels of cytokines and other molecules involved in osteoclastogenesis were assessed in order to determine the inflammatory nature of the disease at a molecular level. The interaction between cytokines and their antagonists will regulate the extent and severity of bone breakdown and tissue destruction mediated by increased levels of IL-1 $1 \beta$, IL-6, TNF- $\alpha$, and RANKL. We found in the diseased tissues, already at 1 and 3-days of ligature placement that periodontal disease significantly upregulated mRNA expression levels of proinflammatory cytokines IL-1 $\beta$, IL-6, and TNF- $\alpha$ compared to the non-ligated animals. IL-1 $\beta$ and IL-6 levels were also elevated 1 week after disease induction but no differences were found 2 and 3 weeks after periodontitis induction for mRNA expression of the cytokines evaluated.

The binding between RANKL to RANK expressed on osteoclast precursors is the primary event for osteoclasts activation. The effects of RANKL are regulated by osteoprotegerin (OPG) that inhibits bone resorption by preventing the interaction of RANK and RANKL (Takayanagi et al., 2002). Alterations in the balance between RANKL and OPG protein expression of define the pathogenesis of several metabolic bone diseases, such as periodontitis. In this context, RANK-L and OPG expression were significantly higher 3 and 5 days after ligature placement. These findings closely resemble observations made by previous studies (de Molon et al., 2014b; de Souza et al., 2011; Matsuda et al., 2015; Saadi-Thiers et al., 2013) in which increased expression of proinflammatory cytokines were noted in the tissue of animals with periodontal disease. 
The increased microbial burden related with the conversion from health status to periodontal disease might also lead to elevated mRNA expression levels of proinflammatory cytokines (de Souza et al., 2011).

An important consideration should be mentioned when interpreting the results of the present investigation. As our focus was mainly in the early stages of disease progression, we evaluated different time points among the analyses. In this regard, histological, radiographic and gene expression of pro-inflammatory cytokines were investigated in the early phase of disease (after 1, 3 and 5 days). Macroscopic, radiographic, and RT-qPCR analyses were also performed in the late stages of disease progression. Histological analysis was not performed in the late stages of disease because there are already available data characterizing the chronic phase of the disease.

\section{CONCLUSIONS}

In summary, our findings in rats provide experimental evidence that ligature-induced periodontal disease offers a consistent model with connective tissue downgrowth, inflammatory cell infiltration, and alveolar bone resorption. Indeed, we showed for the first time, that ligature placement could lead to bone resorption within 24 hours, and two distinct phases were characterized when ligatures are used: an acute phase, with progressive inflammation and bone breakdown, followed by a chronic phase, characterized by absence of significant progression of bone loss. Furthermore, the gene 
expression of IL-6, IL-1 $\beta$, TNF- $\alpha$, RANK-L and OPG were highly increased during the progress of periodontal disease especially in the early periods. 


\section{ACKNOWLEDGEMENTS}

We are extremely grateful to Dr. William V. Giannobile for his help during the experimental design and analysis in this study and for his critical reading of the manuscript.

CONFLICT OF INTEREST

All authors declare that there is no conflict of interest related to this study.

\section{FUNDING:}

The work was supported by the Department of Periodontics and Oral Medicine and Center for Craniofacial Regeneration, School of Dentistry, University of Michigan.

This article is protected by copyright. All rights reserved. 


\section{REFERENCES}

Abe T, Hajishengallis G. 2013. Optimization of the ligature-induced periodontitis model in mice. J Immunol Methods 394(1-2): 49-54.

Albandar JM, Rams TE. 2002. Global epidemiology of periodontal diseases: an overview. Periodontol 2000 29: 7-10.

Alencar VB, Bezerra MM, Lima V, Abreu AL, Brito GA, Rocha FA, Ribeiro RA. 2002. Disodium chlodronate prevents bone resorption in experimental periodontitis in rats. J Periodontol 73(3): 251-6.

Anbinder AL, Moraes RM, Lima GM, Oliveira FE, Campos DR, Rossoni RD, Oliveira LD, Junqueira JC, Ma Y, Elefteriou F. 2016. Periodontal disease exacerbates systemic ovariectomy-induced bone loss in mice. Bone 83: 241-7.

Bezerra MM, Brito GA, Ribeiro RA, Rocha FA. 2002. Low-dose doxycycline prevents inflammatory bone resorption in rats. Braz J Med Biol Res 35(5): 613-6.

This article is protected by copyright. All rights reserved. 
Cavagni J, de Macedo IC, Gaio EJ, Souza A, de Molon RS, Cirelli JA, Hoefel AL, Kucharski LC, Torres IL, Rosing CK. 2016. Obesity and Hyperlipidemia Modulate Alveolar Bone Loss in Wistar Rats. J Periodontol 87(2): e9-e17.

Cirelli JA, Park CH, MacKool K, Taba M, Jr., Lustig KH, Burstein H, Giannobile WV. 2009. AAV2/1-TNFR:Fc gene delivery prevents periodontal disease progression. Gene Ther 16(3): 426-36.

de Aquino SG, Abdollahi-Roodsaz S, Koenders MI, van de Loo FA, Pruijn GJ, Marijnissen RJ, Walgreen B, Helsen MM, van den Bersselaar LA, de Molon RS, Avila Campos MJ, Cunha FQ, Cirelli JA, van den Berg WB. 2014. Periodontal pathogens directly promote autoimmune experimental arthritis by inducing a TLR2- and IL-1-driven Th17 response. J Immunol 192(9): 4103-11.

de Molon RS, Cheong S, Bezouglaia O, Dry SM, Pirih F, Cirelli JA, Aghaloo TL, Tetradis S. 2014a. Spontaneous osteonecrosis of the jaws in the maxilla of mice on antiresorptive treatment: a novel ONJ mouse model. Bone 68: 11-9.

de Molon RS, de Avila ED, Boas Nogueira AV, Chaves de Souza JA, Avila-Campos MJ, de Andrade CR, Cirelli JA. 2014b. Evaluation of the host response in various models of induced periodontal disease in mice. J Periodontol 85(3): 465-77.

de Molon RS, de Avila ED, Cirelli JA. 2013. Host responses induced by different animal models of periodontal disease: a literature review. J Investig Clin Dent 4(4): 2118. 
de Molon RS, Hsu C, Bezouglaia O, Dry SM, Pirih FQ, Soundia A, Cunha FQ, Cirelli JA, Aghaloo TL, Tetradis S. 2016a. Rheumatoid Arthritis Exacerbates the Severity of Osteonecrosis of the Jaws (ONJ) in Mice. A Randomized, Prospective, Controlled Animal Study. J Bone Miner Res 31(8): 1596-607.

de Molon RS, Mascarenhas VI, de Avila ED, Finoti LS, Toffoli GB, Spolidorio DM, Scarel-Caminaga RM, Tetradis S, Cirelli JA. 2016b. Long-term evaluation of oral gavage with periodontopathogens or ligature induction of experimental periodontal disease in mice. Clin Oral Investig 20(6): 1203-16.

de Molon RS, Shimamoto H, Bezouglaia O, Pirih FQ, Dry SM, Kostenuik P, Boyce RW, Dwyer D, Aghaloo TL, Tetradis S. 2015. OPG-Fc but Not Zoledronic Acid Discontinuation Reverses Osteonecrosis of the Jaws (ONJ) in Mice. J Bone Miner Res 30(9): 1627-40.

de Souza JA, Nogueira AV, de Souza PP, Cirelli JA, Garlet GP, Rossa C, Jr. 2011. Expression of suppressor of cytokine signaling 1 and 3 in ligature-induced periodontitis in rats. Arch Oral Biol 56(10): 1120-8.

Eke PI, Dye BA, Wei L, Slade GD, Thornton-Evans GO, Borgnakke WS, Taylor GW, Page RC, Beck JD, Genco RJ. 2015. Update on Prevalence of Periodontitis in Adults in the United States: NHANES 2009 to 2012. J Periodontol 86(5): 611-22.

This article is protected by copyright. All rights reserved. 
Graves DT, Fine D, Teng YT, Van Dyke TE, Hajishengallis G. 2008. The use of rodent models to investigate host-bacteria interactions related to periodontal diseases. $\mathrm{J}$ Clin Periodontol 35(2): 89-105.

Graves DT, Kang J, Andriankaja O, Wada K, Rossa C, Jr. 2012. Animal models to study host-bacteria interactions involved in periodontitis. Front Oral Biol 15: 117-32.

Hiyari S, Atti E, Camargo PM, Eskin E, Lusis AJ, Tetradis S, Pirih FQ. 2015. Heritability of periodontal bone loss in mice. J Periodontal Res 50(6): 730-6.

Hiyari S, Naghibi A, Wong R, Sadreshkevary R, Yi-Ling L, Tetradis S, Camargo PM, Pirih FQ. 2018a. Susceptibility of different mouse strains to peri-implantitis. J Periodontal Res 53(1): 107-116.

Hiyari S, Wong RL, Yaghsezian A, Naghibi A, Tetradis S, Camargo PM, Pirih FQ. 2018b. Ligature-induced peri-implantitis and periodontitis in mice. J Clin Periodontol 45(1): 89-99.

Kilkenny C, Browne WJ, Cuthill IC, Emerson M, Altman DG. 2012. Improving bioscience research reporting: the ARRIVE guidelines for reporting animal research. Osteoarthritis Cartilage 20(4): 256-60.

Klausen B. 1991. Microbiological and immunological aspects of experimental periodontal disease in rats: a review article. J Periodontol 62(1): 59-73.

This article is protected by copyright. All rights reserved. 
Li CH, Amar S. 2007. Morphometric, histomorphometric, and microcomputed tomographic analysis of periodontal inflammatory lesions in a murine model. $\mathrm{J}$ Periodontol 78(6): 1120-8.

Matsuda Y, Kato T, Takahashi N, Nakajima M, Arimatsu K, Minagawa T, Sato K, Ohno H, Yamazaki K. 2015. Ligature-induced periodontitis in mice induces elevated levels of circulating interleukin-6 but shows only weak effects on adipose and liver tissues. J Periodontal Res.

Mizuno M, Miyazawa K, Tabuchi M, Tanaka M, Yoshizako M, Minamoto C, Torii Y, Tamaoka Y, Kawatani M, Osada H, Maeda H, Goto S. 2015. Reveromycin A Administration Prevents Alveolar Bone Loss in Osteoprotegerin Knockout Mice with Periodontal Disease. Sci Rep 5: 16510.

Mundy GR. 1991. Inflammatory mediators and the destruction of bone. J Periodontal Res 26(3 Pt 2): 213-7.

Nogueira AV, de Molon RS, Nokhbehsaim M, Deschner J, Cirelli JA. 2017. Contribution of biomechanical forces to inflammation-induced bone resorption. $\mathrm{J}$ Clin Periodontol 44(1): 31-41.

Nogueira AV, de Souza JA, de Molon RS, Pereira Eda S, de Aquino SG, Giannobile WV, Cirelli JA. 2014. HMGB1 localization during experimental periodontitis. Mediators Inflamm 2014: 816320.

This article is protected by copyright. All rights reserved. 
Pirih FQ, Hiyari S, Barroso AD, Jorge AC, Perussolo J, Atti E, Tetradis S, Camargo PM. 2015. Ligature-induced peri-implantitis in mice. J Periodontal Res 50(4): 519-24.

Polak D, Shapira L, Weiss EI, Houri-Haddad Y. 2013. Virulence mechanism of bacteria in mixed infection: attenuation of cytokine levels and evasion of polymorphonuclear leukocyte phagocytosis. J Periodontol 84(10): 1463-8.

Polak D, Wilensky A, Shapira L, Halabi A, Goldstein D, Weiss EI, Houri-Haddad Y. 2009. Mouse model of experimental periodontitis induced by Porphyromonas gingivalis/Fusobacterium nucleatum infection: bone loss and host response. J Clin Periodontol 36(5): 406-10.

Saadi-Thiers K, Huck O, Simonis P, Tilly P, Fabre JE, Tenenbaum H, Davideau JL. 2013. Periodontal and systemic responses in various mice models of experimental periodontitis: respective roles of inflammation duration and Porphyromonas gingivalis infection. J Periodontol 84(3): 396-406.

Takayanagi H, Kim S, Matsuo K, Suzuki H, Suzuki T, Sato K, Yokochi T, Oda H, Nakamura K, Ida N, Wagner EF, Taniguchi T. 2002. RANKL maintains bone homeostasis through c-Fos-dependent induction of interferon-beta. Nature 416(6882): 744-9.

Wilensky A, Gabet Y, Yumoto H, Houri-Haddad Y, Shapira L. 2005. Three-dimensional quantification of alveolar bone loss in Porphyromonas gingivalis-infected mice using micro-computed tomography. J Periodontol 76(8): 1282-6.

This article is protected by copyright. All rights reserved. 
Wong RL, Hiyari S, Yaghsezian A, Davar M, Lin YL, Galvan M, Tetradis S, Camargo PM, Pirih FQ. 2017. Comparing the Healing Potential of Late-Stage Periodontitis and Peri-Implantitis. J Oral Implantol 43(6): 437-445.

Yuan H, Gupte R, Zelkha S, Amar S. 2011. Receptor activator of nuclear factor kappa B ligand antagonists inhibit tissue inflammation and bone loss in experimental periodontitis. J Clin Periodontol 38(11): 1029-36.

This article is protected by copyright. All rights reserved. 


\section{FIGURE LEGENDS}

Figure 1. A - Schematic representation of ligature placement around the $1^{\text {st }}$ and $2^{\text {nd }}$ maxillary molars in an "8” shape and knotted at the palatal surface of the second molar. Ligatures were kept in position during the entire experimental period and were checked every other day and repositioned if necessary. B-C - Macroscopic views of the molars showing the ligature in placement after (B) soft tissue removal, and the alveolar bone loss induced by the ligature around both molars (C).

Figure 2. A-D - Representative samples stained with methylene blue in a palatal (A, C, E, G) and buccal (B, D, F, H) view at baseline and after 7, 14 and 21 days of ligature placement. Digital images of the buccal and palatal surfaces of the samples were obtained with a stereomicroscope with 20x magnification. The distance from the CEJ to the ABC was measured at 6 different sites per tooth (mesio-distal, disto-mesial, buccal-palatal and palatal-buccal regions) and mean values were used. I - Linear measurements in the distal 
area of the $1^{\text {st }}$ molar, mesial aspect of the $2^{\text {nd }}$ molar and within the interproximal area. ${ }^{\dagger}$ Statistically significantly different, $\mathrm{p}<0.001$. ${ }^{*}$ Statistically significantly different, $\mathrm{p}<$ 0.0001. Data represent the mean \pm SD.

Figure 3. Representative $3 \mathrm{D}$ images showing in detail the linear measurements performed for each sample. The distance between the cemento-enamel junction (CEJ) to the alveolar bone crest $(\mathrm{ABC})$ was measured in the mesial region of the first molar and distal region of the second molar.

Figure 4. Quantification of the $\mu \mathrm{CT}$ linear measurements. Linear bone loss evaluation in the mesial aspect of the first molar (A), distal aspect of the second molar (B) and interproximal area between the first and the second molars (C). The distance from the CEJ to the ABC was measured at the palatal surface of the mesio- and disto-palatal roots of the maxillary $1^{\text {st }}$ and $2^{\text {nd }}$ molar teeth. Characterization of the two different phases of the disease (D). * Statistically significantly different, $\mathrm{p}<0.05$. Data represent the mean \pm SD.

Figure 5. Representative histological sections of the experimental groups after periodontitis induction at 1,3 , and 5 days $(A, D, G)$ within the furcation region $(B, E, H)$ and in the interproximal (C, F, I) areas. Samples were paraffin-embedded and $5 \mu \mathrm{m}$-thick 
sections were obtained in the mesio-distal direction and were stained with $\mathrm{H} \& \mathrm{E}$ for descriptive histologic analysis. Black arrows point to inflammatory infiltrate in the connective tissue and the blue arrows point to the alveolar bone crest.

Figure 6. qPCR for mRNA expression during the course of the experimental periodontal disease for proinflammatory cytokines IL-6 (A, B), IL-1 $(\mathrm{C}, \mathrm{D})$ and TNF- $\alpha$ (E, F), and proteins involved in osteoclastogenesis RANKL $(\mathrm{G})$ and OPG $(\mathrm{H})$, and the ratio between RANK-L and OPG (I). Gene expression levels were normalized to the housekeeping gene, GAPDH. ${ }^{*}$ Statistically significant difference $=\mathrm{p}<0.05 .{ }^{* *}$ Statistically significant difference $=\mathrm{p}<0.01$. Data are represented as the mean values \pm SD.

\section{Table Legends}

Table 1. Inventoried TaqMan Primers and probe. 

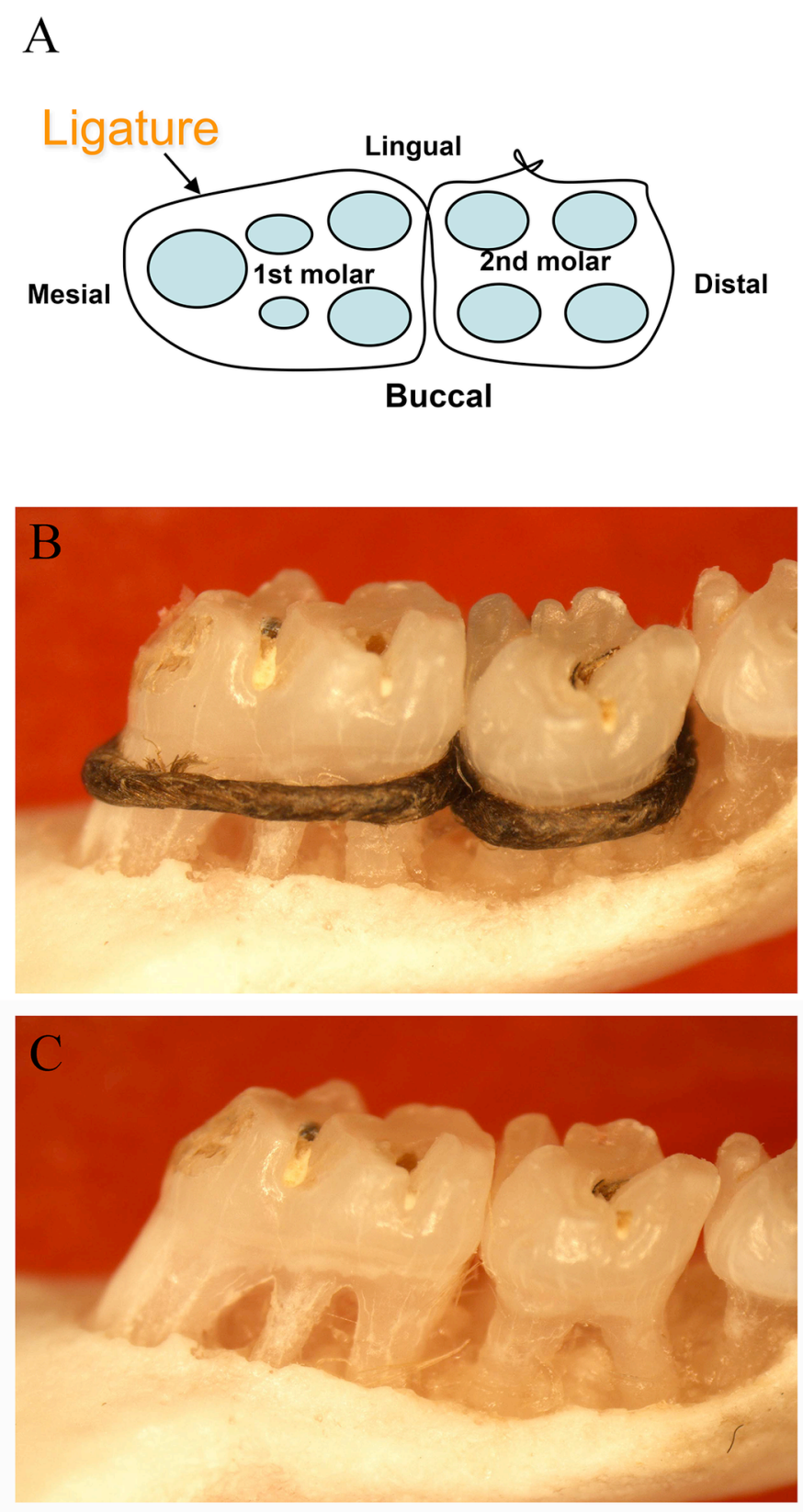

Figure 1.tif

This article is protected by copyright. All rights reserved. 


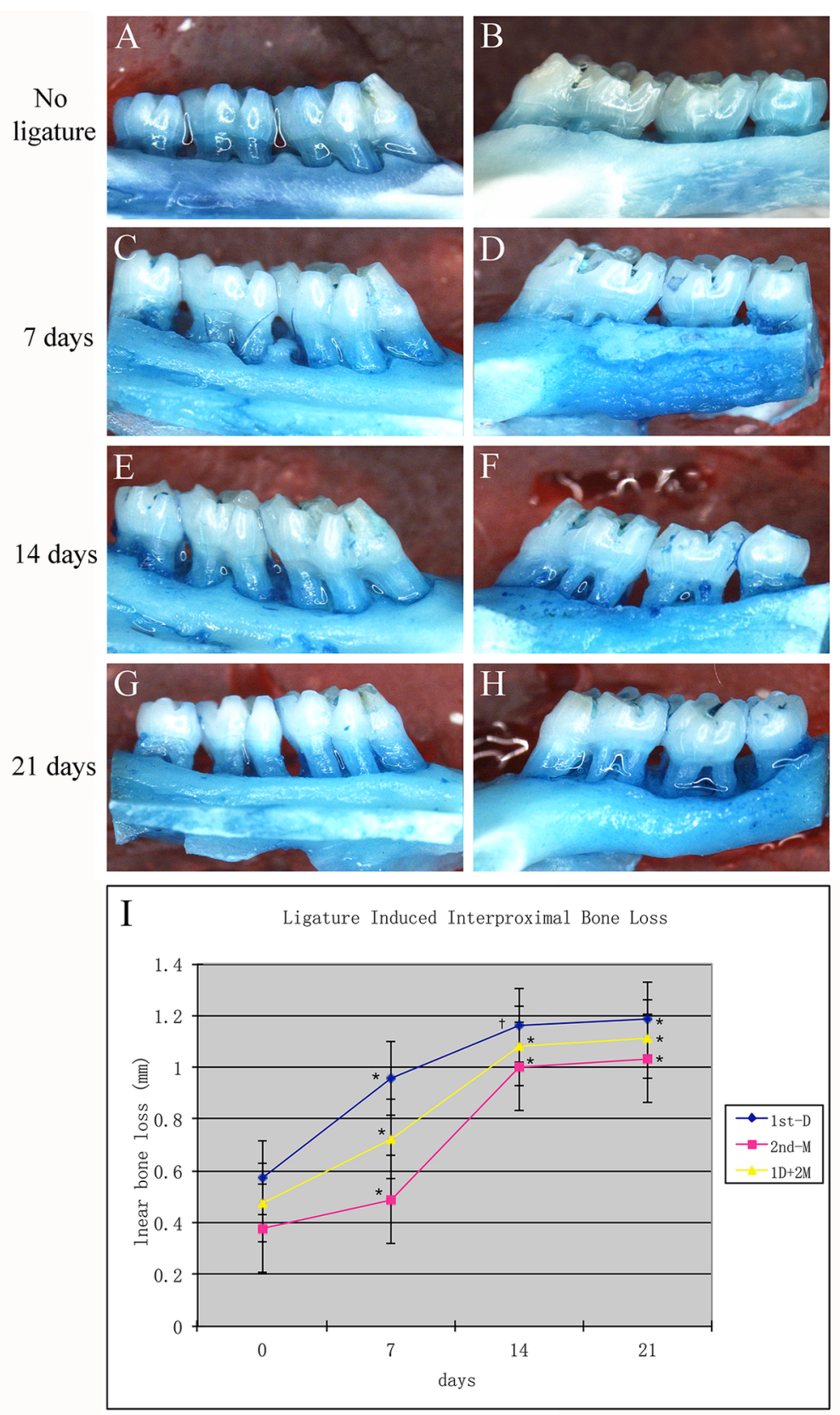

Figure 2.tif 

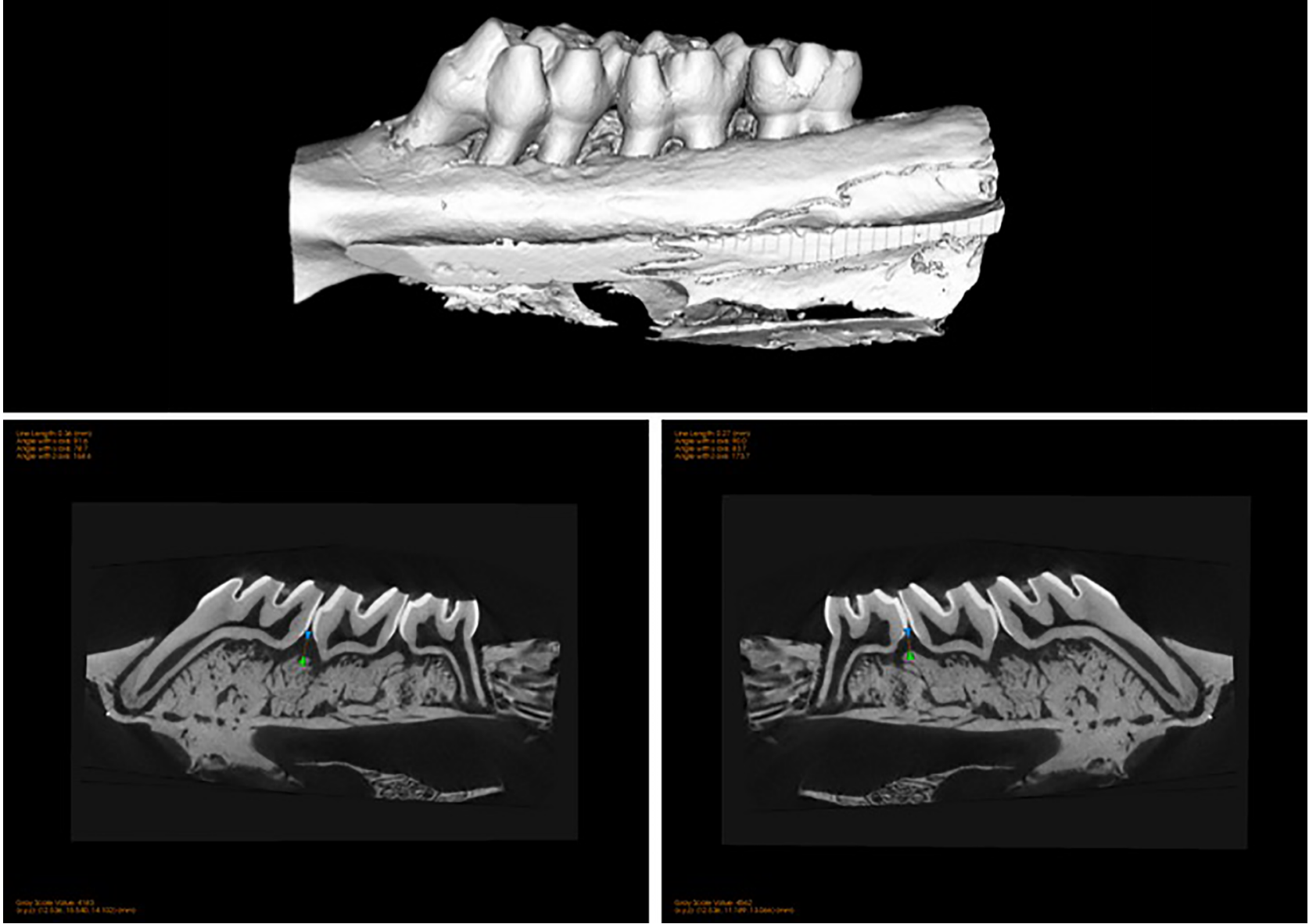

Figure 3.tif

This article is protected by copyright. All rights reserved. 
A

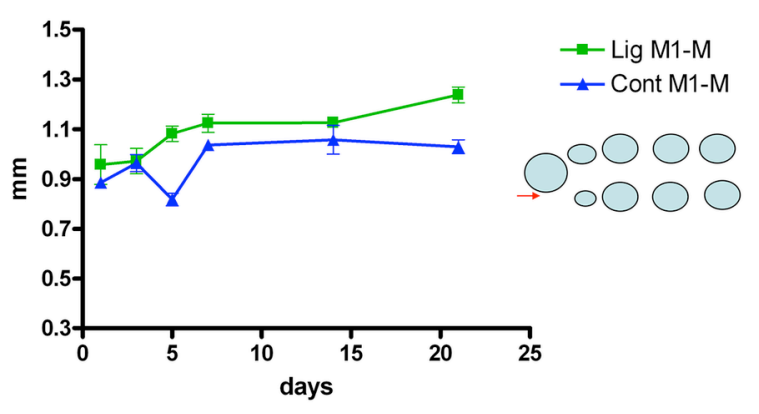

C

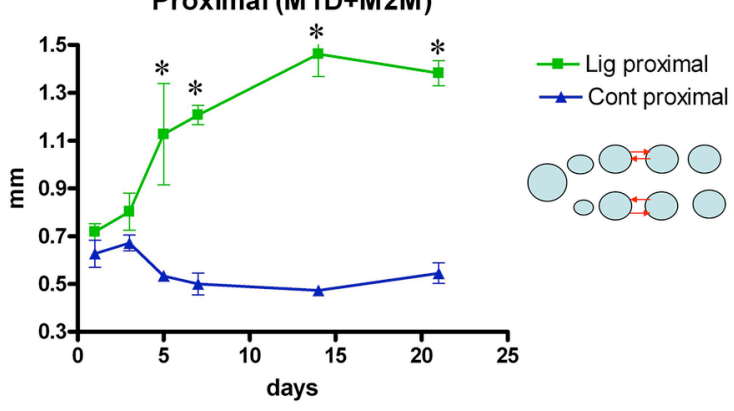

B Distal M2

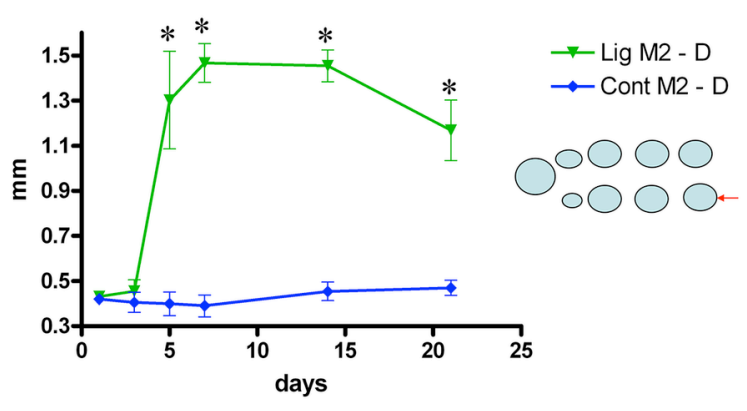

$\mathrm{D}$

Interproximal bone loss

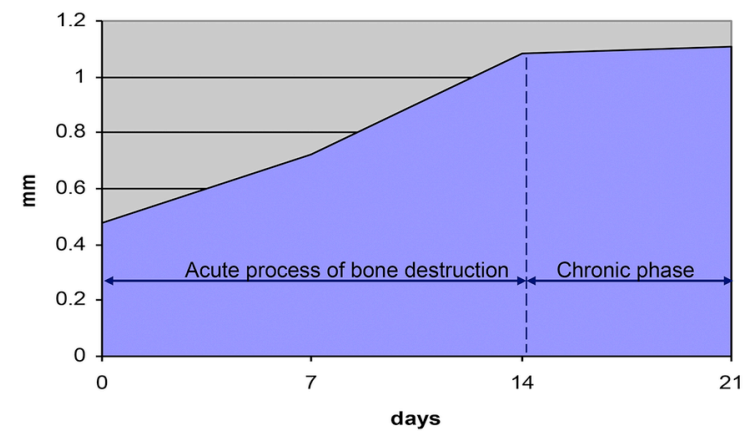

Figure 4.tif

This article is protected by copyright. All rights reserved. 


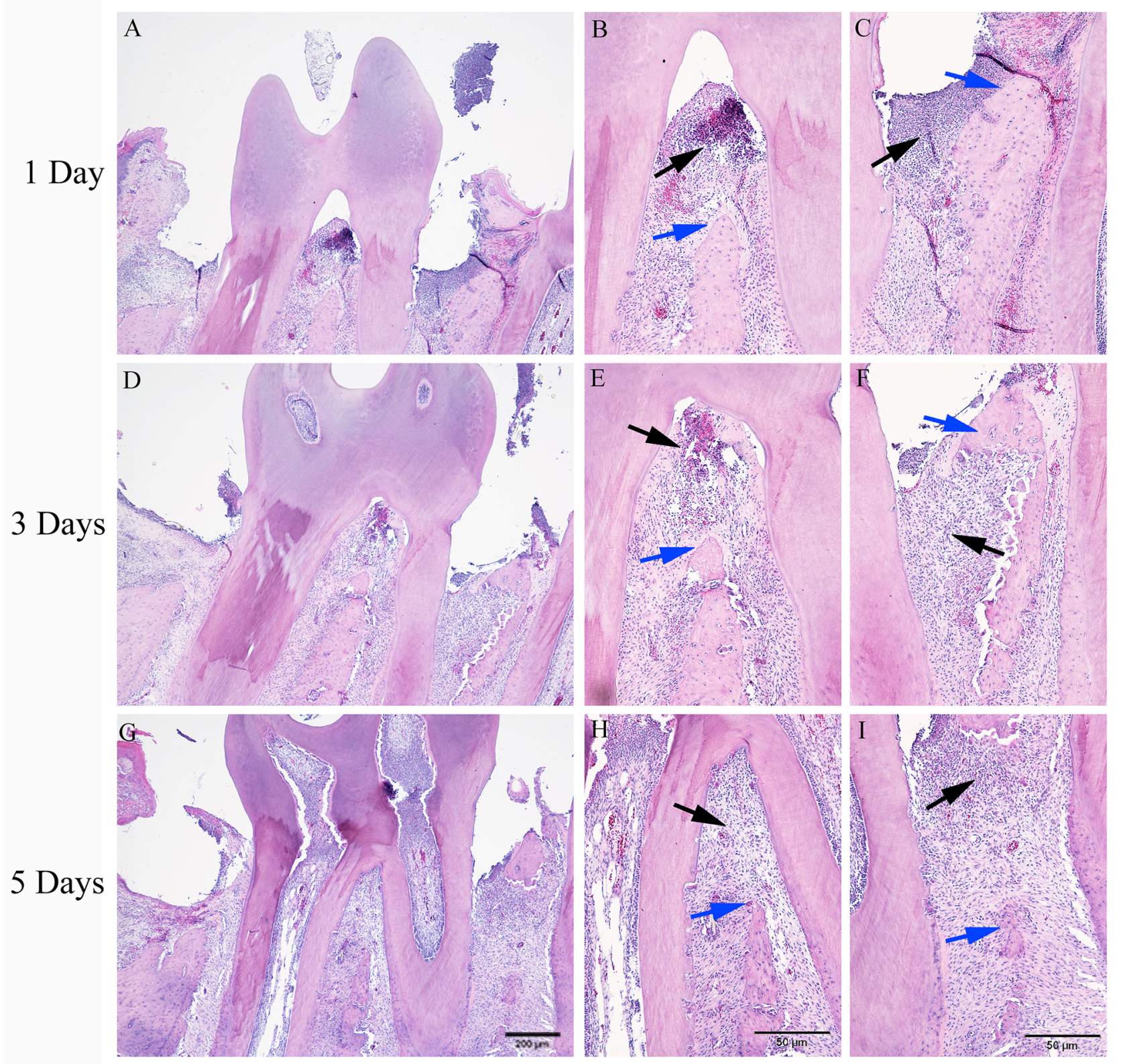

Figure 5.tif 

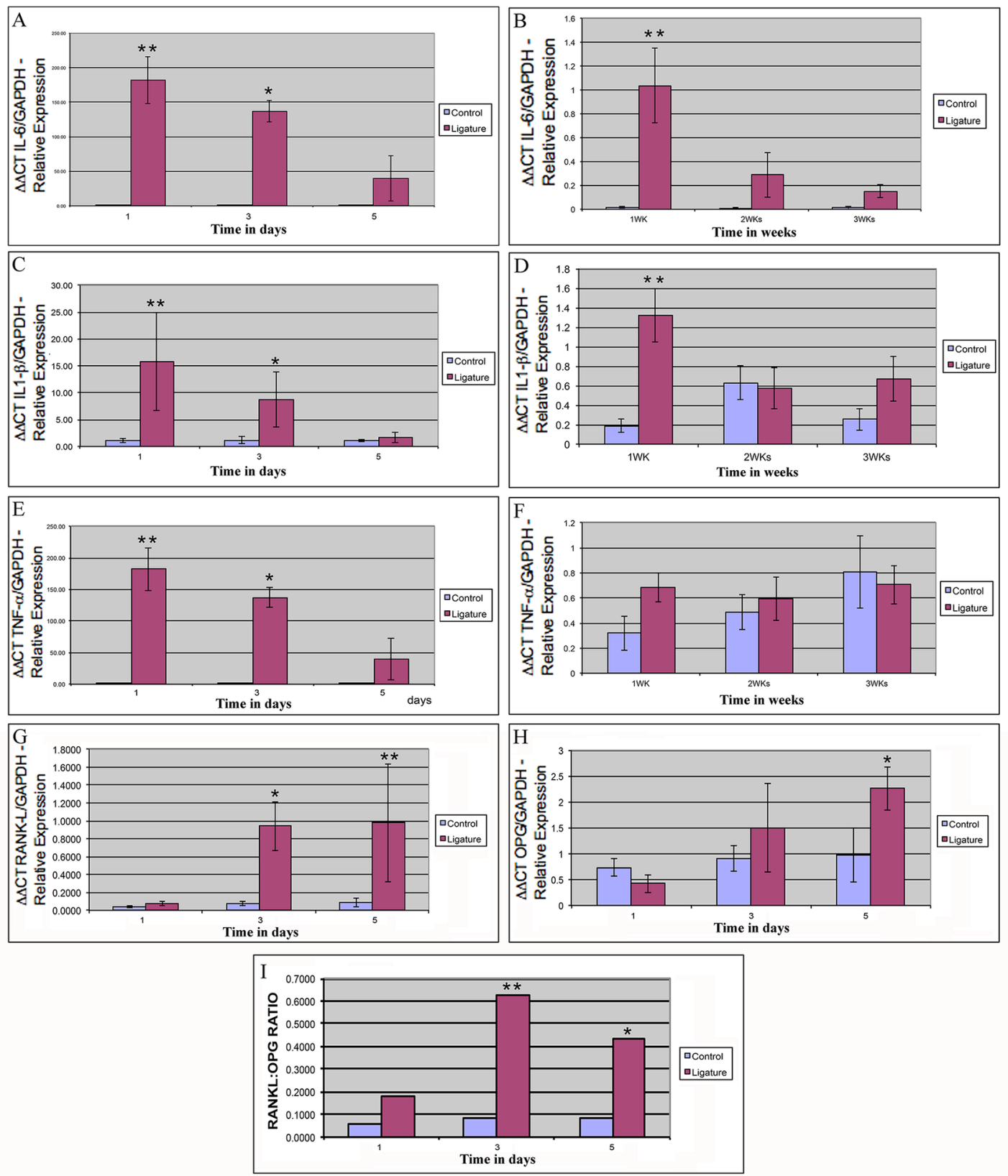

Figure 6.tif 

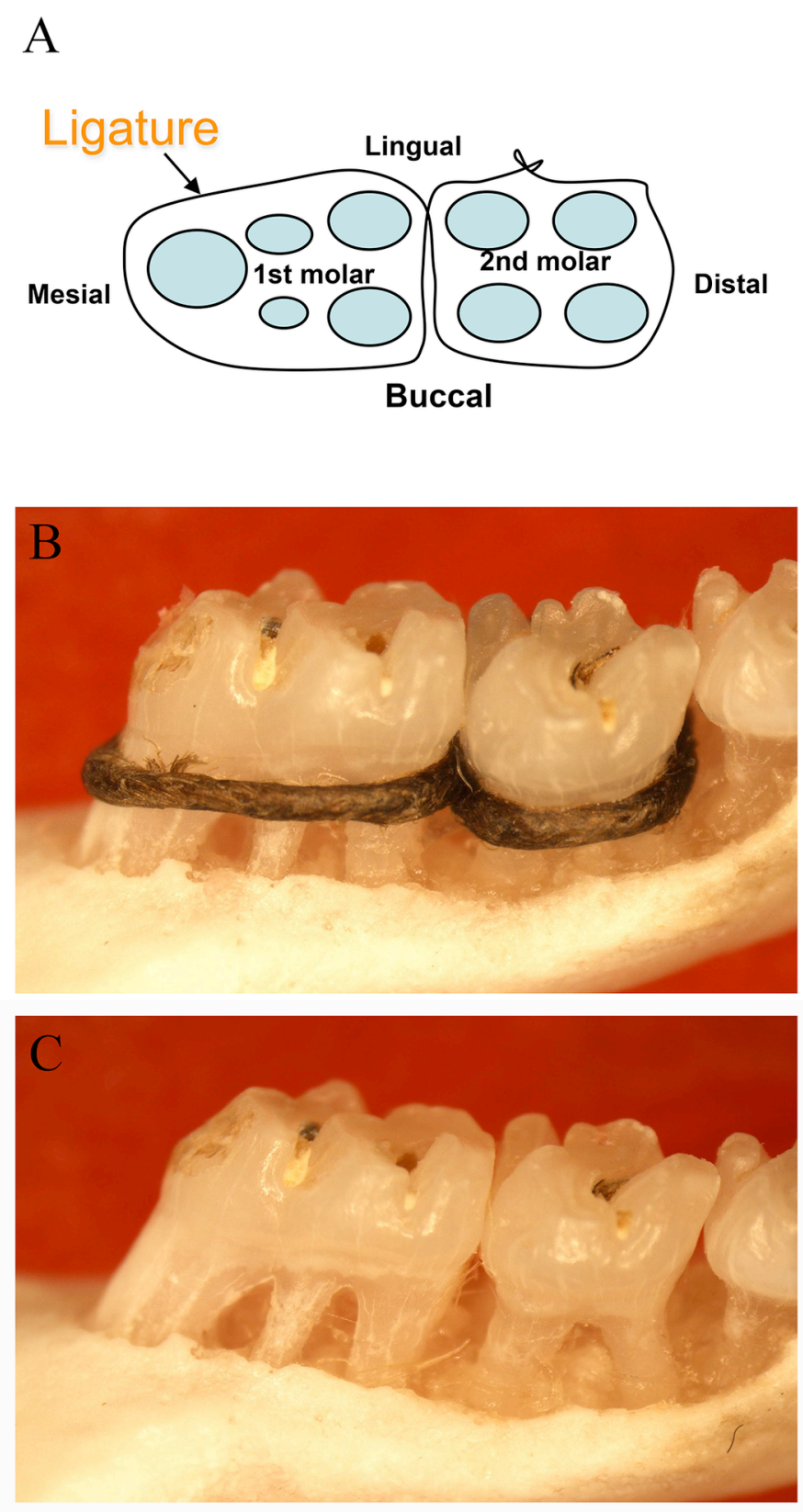

Graphical Abstract.tif

This article is protected by copyright. All rights reserved. 
Table 1 - Inventoried TaqMan Primers and probe.

\begin{tabular}{lcc}
\hline Target Gene & ABI ID no & Reporter Probe Sequence \\
\hline IL $-1 \beta$ & Rn00580432_m1 & CATAAGCCAACAAGTGGTATTCTCC \\
IL-6 & Rn00561420_m1 & GAGAAAAGAGTTGTGCAATGGCAAT \\
TNF- $\alpha$ & Rn99999017_m1 & CACACTCAGATCATCTTCTCAAAAC \\
RANK-L & Rn00589289_m1 & TGCCGACATCCCATCGGGTTCCCAT \\
OPG & Rn00563499_m1 & GCTGTGCACTCCTGGTGTTCTTGGA \\
\hline
\end{tabular}

This article is protected by copyright. All rights reserved. 